\title{
PARTICULARS OF ADSORBENT REGENERATION WITH THE USE OF MICROWAVE ENERGY
}

\author{
Sergey Dobrotvorskiy*, Ludmila Dobrovolska, Yevhenita Basova, \\ BORYS ALEKSENKO
}

\author{
National Technical University "Kharkov Polytechnic Institute", 2, Kyrpychova str., Kharkov, Ukraine \\ * corresponding author: sdobro50@gmail.com
}

\begin{abstract}
Energy conservation issues are acute in the world. Compressed air is widely used in the modern industrial production. The production of compressed air is a very energy-intensive process, since most of the energy, which is expended by the compressor, passes into the energy of heating. Compressed air cannot be used in modern production without a prior drying and cleaning. Industrial dryer's air losses is up to $20 \%$ of compressed air additionally. Therefore, the issue of saving air during its drying stage is important.

In the presented article, the thermal and aerodynamic processes that occur in the classical adsorption tower with the most modern design are considered. The processes that occur in the adsorption column with the microwave regeneration of the adsorbent are also considered. A comparative analysis of these constructions from the point of view of energy saving is made.
\end{abstract}

KEYWORDS: dryer; microwave radiation; regeneration; computer model.

\section{InTRODUCTION}

Compressed air dryers with a hot regeneration of the adsorbent are currently the most economical means of producing dried air. Developing the design for this type of equipment is the most important direction to improve the energy efficiency of industrial production. The way of increasing the efficiency of parts manufacturing, production planning stage and fixture design is an urgent task [1 3 .

The main attention of developers of drying equipment with the hot regeneration is focused on the problems of the heat energy recovery, which is used in the desorption process.

The design of the adsorption dryer should provide the maximum possible energy conservation aimed at regenerating the adsorbent. In the case the regeneration uses the method of hot regeneration, it is desirable to reduce the level of thermal energy losses [4, 5].

Thermal energy is carried by purge air. The purge air is preheated using a heating element and then moves into the cavity of the adsorption column, where it releases the energy during the interaction with the volume of the adsorbent.

With this method of heat transfer, the purge air loses a large portion of the energy in the path of the hot air from the heating element to the cavity of the adsorption column. This is due to the fact that the walls of the the air ducts are in contact with the heated air on one side and the environment on the other side. This implies the presence of a convective heat transfer between the heated air and the duct wall and a similar heat exchange between the duct wall and the surrounding environment. Since the purge air heats up to a temperature of $+200{ }^{\circ} \mathrm{C}$, and the ambient temperature is, on average, $+20^{\circ} \mathrm{C}$, the temperature difference is about $180^{\circ} \mathrm{C}$. The thermal insulation of the air ducts, which is used by the manufacturers in the design of their air dryers, can partially solve the problem of the loss of thermal energy.

A disadvantage of this method of increasing the energy efficiency level of the desiccant is that the metal parts of the desiccant inevitably provide a mutual heat exchange between themselves. Since it is not possible to perform a complete thermal insulation of the entire air dryer, the loss of thermal energy occurs through the components of the dryer, which are not sufficiently insulated and act as a radiator. In addition, in accordance with the dryer's working cycle, the heater operates periodically [6]. During the heating of the air, the heating element of the heater has a temperature of $+200^{\circ} \mathrm{C}$ [7. After that, when the heater switches off, the heating element begins to cool, continuing to unproductively transfer the heat energy to the environment.

At present, the most modern, most economically feasible and efficient design solution is the placement of the heating element directly in the cavity of the adsorption column [8]. In this way, designers solve the problem of an unproductive heat loss, avoiding the need to transport the heated air from the heater to the adsorption column. This implies the absence of an air duct and the need to ensure its thermal insulation.

Due to its advantages in terms of energy efficiency, this design of the adsorption column (hereinafter referred to as "HT adsorption column") has found a practical application in the construction of adsorption dryers with the hot regeneration. The placement of the heater in the cavity of the adsorption column also causes the simplification of the design and the 


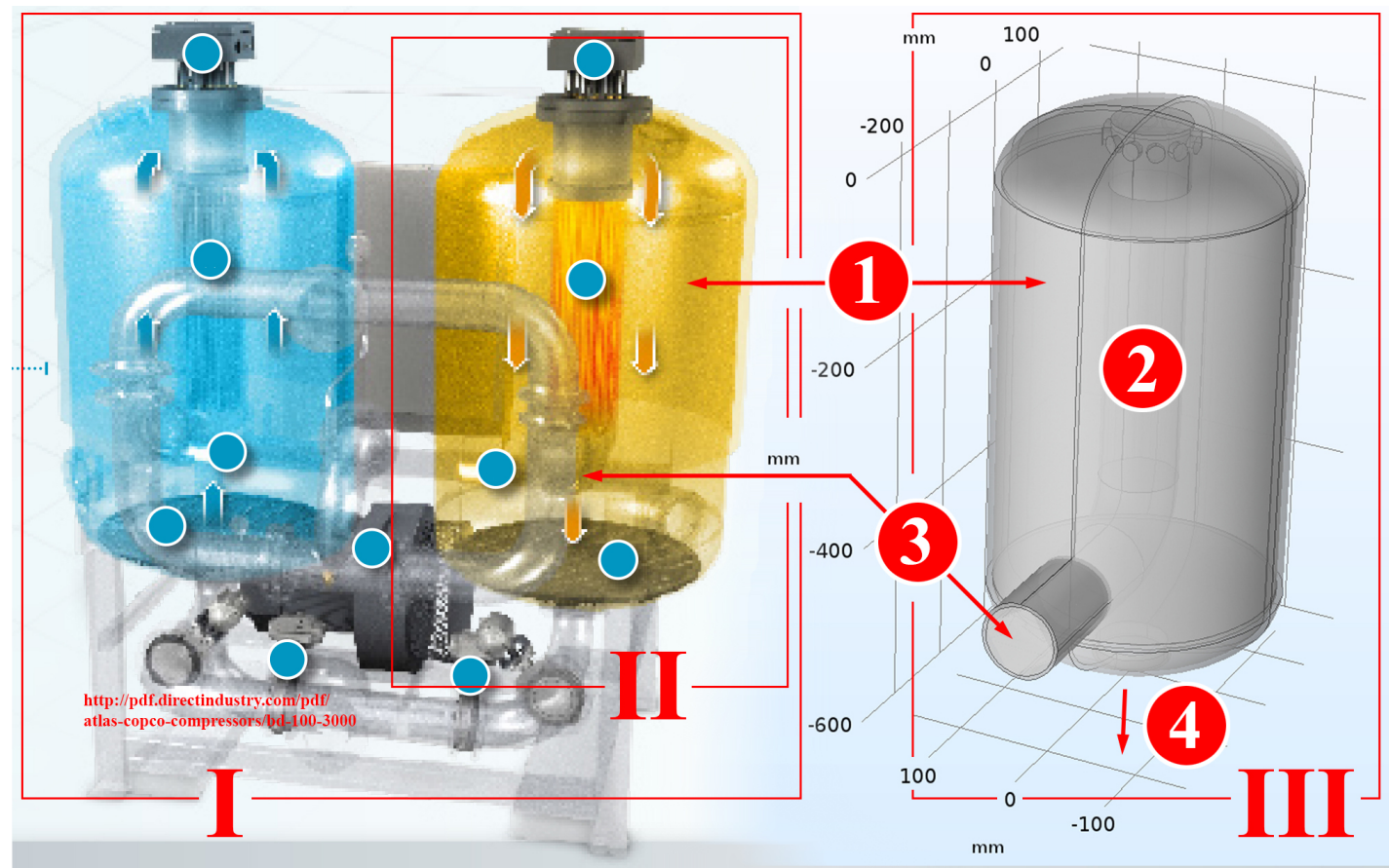

FiguRE 1. Adsorption dryer [8] with hot regeneration of adsorbent (I), adsorption column with the built-in heater in the regeneration stage (II) and the computer model of this column (III): 1 is the body of adsorption column; 2 is the internal heater; 3 is the inlet of the non-heated dry purge air; 4 is the output of the heated, moisture-saturated purge air.

reduction in the number of parts of the dryer. At the same time, maintainability and serviceability of the air dryer are kept high.

In this regard, it is important to note the technology of hot regeneration, which allows removing water molecules from the porous structure, reducing the energy impact on the absorbent. At the same time, the electric heater is excluded from the construction of the column and the need to transport the heat from the heater to the adsorbent is eliminated. This is possible in the case of using the energy of the microwave radiation [5, 11, 25].

\section{RESEARCH PROBLEM}

The design of the air dryer with the HT adsorption columns should be further studied in terms of the presence of its disadvantages. These drawbacks may stem from the new principle of organizing the process of heating the volume of the adsorbent in the cavity of the HT adsorption column and, therefore, to be constructively irremovable without seriously changing this new principle.

From the point of view of efficiency and expediency of using alternative types of energy in the process of regenerating the adsorbent in adsorption dryers of compressed air with HT columns, the disadvantages of this design of the adsorption column should be determined by a comparative analysis of those processes that occur during the regeneration period. From the point of view of changing the temperature of the adsorbent during the hot regeneration, this process is carried out in two stages. In the first stage, during the purging with the heated air, the temperature of the adsorbent rises to the temperature of the purge air.

The first stage of regeneration is continued until the moisture content in the adsorbent reaches a predetermined value. The duration of the first stage of the regeneration process decreases in the case of an increase in the rate of the removal of the moisture from the volume of the adsorbent. In the second stage, the volume of the adsorbent is cooled to a temperature not exceeding the temperature of the drying air. The duration of this stage also affects the duration of the regeneration process. The second stage of regeneration should not affect the moisture content of the adsorbent dried in the first stage. Cooling the adsorbent to the temperature of the dried compressed air is the final stage of the regeneration process. This is an essential condition for the subsequent adsorption process since the hot adsorbent [9, 10, is not capable of actively adsorbing the moisture from the dried air.

And, as it was pointed out in [11, increasing the efficiency of the regeneration process should not lead to an increase in the duration of the regeneration period beyond the duration of the adsorption period, since this leads to a need for more complicated design of the dryer and the use of additional adsorption columns to ensure the continuity of the desiccation.

In the present work, thermal processes that occur in the cavity of the HT adsorption column are investigated. Namely the generation, transfer and heat transfer that are crucial from the point of view of the 


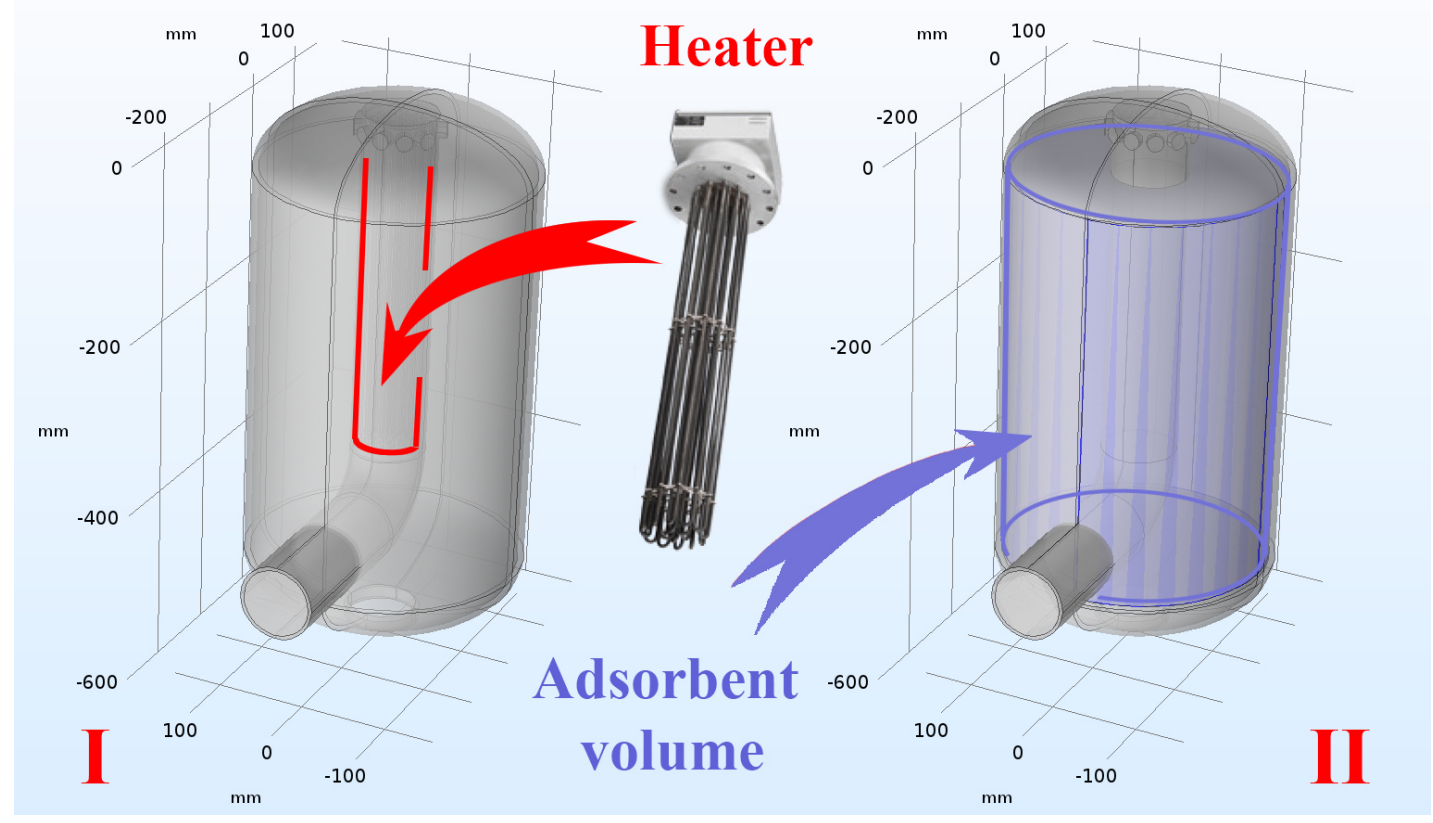

Figure 2. Column model with the built-in heater: I - location of the internal heat source (heater); II - placement of adsorbent in the cavity of the column.

efficiency of moisture removal from the adsorbent by the hot regeneration method.

Today, the study of the course of various processes of heat transfer and phase transformations are done using computer models 12 15. The study of the HT adsorption column of a modern design, which is practically used in industry, is carried out using a computer simulation by constructing an adsorption column model (Figure 1), and studies of the processes that occur in it. Also, computer models of the HT adsorption column and the adsorption column, which uses microwave energy in the process of regeneration (hereinafter referred to as "RF adsorption column"), are compared.

The study uses computer models of adsorption columns with a capacity of $5 \mathrm{~m}^{3} / \mathrm{min}$. The HT adsorption column model has an internal heat source that physically and geometrically repeats the heater installed in the cavity of the adsorption column. Channels through which air flows, the heater chamber and the volume of adsorbent in the column cavity are also modelled (Figure 2).

\section{MAterials AND METHOdS}

The adsorption dryer column operates cyclically (here, hot regeneration is considered). Initially, compressed moist air passes through the column and the adsorbent absorbs moisture from it. This period lasts 4 hours. Then, the adsorbent should be dried. To do this, its temperature must be raised to $200{ }^{\circ} \mathrm{C}$, and the purge air with atmospheric pressure passes through to the adsorbent. The purge air transfers moisture to the atmosphere. Usually, the hot drying stage lasts 2 hours. The regenerated adsorbent is ready to dry the compressed air again. However, the adsorption process is not possible at a high temperature of the adsorbent. In order to cool the hot adsorbent, it is needed to blow it with cold dry air. If the dryer includes 2 adsorption columns, then, for its continuous operation, the duration of the regeneration period of the adsorbent should not exceed 4 hours. Therefore, the cooling stage should not last longer than 2 hours. The design of the adsorption column should provide a rapid heating of the adsorbent and its rapid cooling with a minimal dry cooling air consumption.

In the process of solving the model built in COMSOL Multiphysics, the following equations are used. The stationary problem of the air medium's motion was calculated by the formula:

$$
\begin{gathered}
\rho(u \cdot \nabla) \cdot u=\nabla \times\left(-p l+\mu\left(\nabla u+(\nabla u)^{T}\right)\right. \\
\left.-\frac{2}{3} \mu(\nabla \cdot u) l\right)+F, \\
\nabla \cdot(\rho u)=0 .
\end{gathered}
$$

where $u$ is the velocity, $p$ is the pressure, $\mu$ is the dynamic viscosity.

The movement of air in the volume of the adsorbent was modelled as a motion in a porous medium and was calculated by the formula:

$$
\begin{gathered}
\frac{1}{\epsilon_{p}} \rho(u \cdot \nabla) u \frac{1}{\epsilon_{p}} \nabla\left[-p l+\mu \frac{1}{\epsilon_{p}}\left(\nabla u+(\nabla u)^{T}\right)\right. \\
\left.-\frac{2}{3} \mu \frac{1}{\epsilon_{p}}(\nabla \cdot u) l\right]-\left(\mu k^{-1}+\beta_{F}|u| \frac{Q_{m}}{\epsilon_{p}^{2}}\right) u+F, \\
\nabla \cdot(\rho u)=Q_{m}
\end{gathered}
$$

where $Q_{m}$ is the value of porosity of the material.

The dynamic heat transfer problem was calculated 


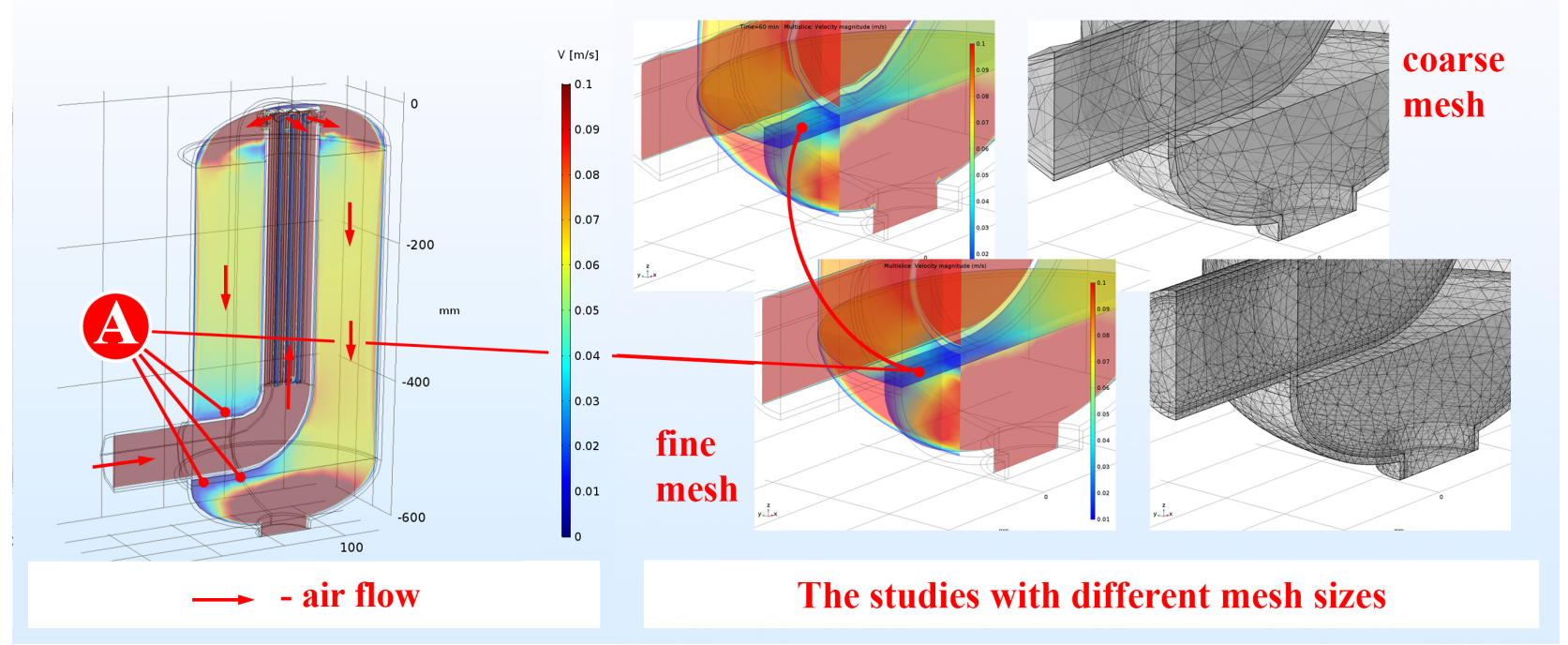

FIGURE 3. The gradient of the rate of airflow in the cavity of the adsorption column: A is the zone with a low speed of air movement, which is within the limits of the volume of the adsorbent.

by the formula:

$$
\begin{gathered}
\rho C_{p} u \cdot \nabla T+\nabla \cdot q=Q+Q_{v d} \\
q=-k_{e f f} \nabla T
\end{gathered}
$$

where $C_{p}$ is the heat capacity value, $T$ is the temperature, $Q$ is the quantity of heat.

Heat transfer in a porous medium was calculated as

$$
k_{e f f}=\Theta_{p} k_{p}+\left(1-\Theta_{p}\right) k+k_{d i s p}
$$

where $\Theta_{p}$ is the volume fraction of the material, $k_{p}$ is the thermal conductivity [16].

Adsorbents have a different porosity at a scale of a separate granule. This defines the adsorption capacity and affects the intensity of the regeneration process. The gas-dynamic process, considered at a scale of the adsorption column, will be determined by the size and shape of the adsorbent granules. This defines the bulk density of the adsorbent volume. The adsorbents, used in dryers, have the same characteristics: adsorbent granules have a spherical shape and a size of $3-7 \mathrm{~mm}$. The normal range of porosities in granular systems is $5 \%$ to $35 \%$. A hexagonal or cubic close pattern is assumed, the calculated packing density is 0.7045 [26], giving a porosity void fraction of 0.2955 , provided that all the balls are the same size. The difference in grain size will create a lower porosity. But adsorbent retaining grids of modern dryers have the ability of removing small granules and products of their shredding. This makes it possible to consider the porosity of the adsorbent volume to be $30 \%$. Granules will not give closed pores, so the effective porosity is considered equal to $100 \%$.

The input parameters of the air movement are given through the velocity $U_{0}=1 \mathrm{~m} / \mathrm{s}$ of the flow at the input (Figure 1. item 3). The output parameters are defined as a free boundary with zero pressure $P_{o}=0 \mathrm{~kg} / \mathrm{s} \mathrm{m}^{2}$.

In the process of studying the heat transfer processes that occur in the column during its heating using an internal heater, the input parameters were set to the same $T_{0}=+20^{\circ} \mathrm{C}$ for all domains of the components of the adsorption column, with the exception of the heater domain. The heater temperature $T_{0}=+210^{\circ} \mathrm{C}$ is set separately. It is assumed that the entire volume of the adsorbent and all components of the adsorption column initially have the same temperature, equal to $+20^{\circ} \mathrm{C}$. The difference in temperature in the adsorbent layers, due to the unequal release of the heat of adsorption, is neglected as insignificant, compared to the intensity of conductive heat transfer inside the volume of the adsorbent and convective heat transfer from the adsorbent to the purge air.

In the process of studying the heat transfer processes that occur in the column during its cooling by the flowing purge air, the input parameters of the heat transfer model are given by the same $T_{0}=+200{ }^{\circ} \mathrm{C}$ for all domains when modelling the cooling process of the column. It is assumed that the entire volume of the adsorbent is evenly heated, and all components of the adsorption column also have a temperature equal to the temperature of the adsorbent. Thermal losses through the wall of the adsorption column to the environment are neglected as insignificant, compared to the intensity of heat transfer from the adsorbent to the purge air.

It is assumed that the influence of the transient processes that occur in the adsorption column during the change of the regeneration stage to the adsorption stage and back is negligible in this study. This is because the transients are fleeting $(5-15 \mathrm{~s})$, compared to the duration of the hot regeneration cycle (4-8h), and do not have the time to have a significant effect on the processes that occur in the adsorption 


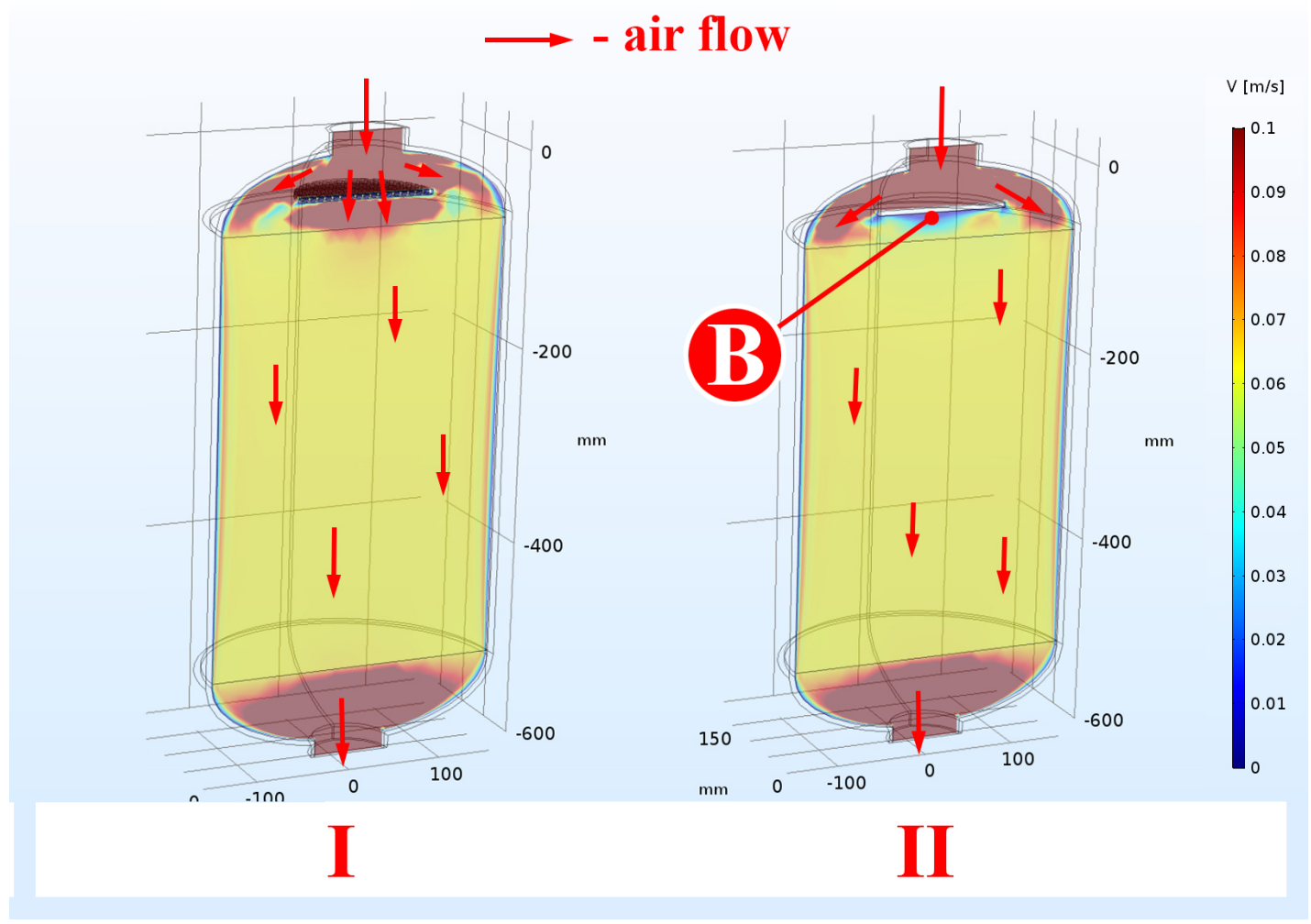

FiguRE 4. The gradient of the rate of airflow in the cavity of the adsorption column RF: I is the lattice diffuser; II is the baffle plate. B is a zone with a low air velocity that is placed outside the boundaries of the volume of the adsorbent.

column. Therefore, the solution of the flux pattern of the air flow in the adsorption column and through the adsorbent bed that occurs during the desorption stage is performed using a stationary solver. In the process of solution, data were obtained on the flow rate and pressure of the medium. Since the air flow speed in the adsorption column with hot regeneration should not exceed $0.2 \mathrm{~m} / \mathrm{s}$, the problem of studying turbulent flows at such a speed of air movement is not set now. The PARDISO solver was used as the fastest and least resource intensive. To verify the results, the model was calculated with a different grid size (Figure 3), herewith the results of the calculations do not fundamentally diverge from one to another.

The processes of heat transfer in the air and porous media, as well as in the steel components of the adsorption column casing, are modelled and solved using a time-dependent solver. It gives a possibility to trace the thermal processes occurring in the column in dynamics.

Based on the data obtained, gradients of speed and temperature of the studied processes, graphs of dependence and comparative graphs are constructed.

\section{Results AND Discussion}

\subsection{Airflow in the adsorption COlumn}

Since the bulk of the heat energy in the process of regeneration is transmitted by purge air, the flow characteristics in the cavity of the adsorption column strongly affect the thermal process [17]. The study begins with the calculation of the flow in the cavity of adsorption columns.

\subsubsection{Airflow in the adsorption COLUmN HT}

The air entering the adsorption column HT enters the heater chamber. Then, from the heater chamber, air enters directly into the cavity of the adsorption column containing the adsorbent. Air moves through the volume of the adsorbent, which is a porous medium, from top to bottom (Figure 3). The direction of the purge air movement from top to bottom is adopted in most designs of dryers and is the most acceptable from the point of view of the reliability of the obtaining guaranteed dehydrated compressed air [18, at the exit from the air dryer. After passing through the volume of the adsorbent, the purge air freely leaves the adsorption column.

The data on the maximum, minimum, and average flow rates of the air flow through the HT adsorption column and through the adsorbent bed (Figure 3) indicate the presence of zones with a low (up to $0.01 \mathrm{~m} / \mathrm{s}$ ) air velocity. During the consideration of the velocity gradient, zones with a low air velocity within the adsorbent volume located near the inlet duct and near the heater chamber are detected (Figure 3 item I, letter A.) Since the drying rate of the adsorbent during the drying phase depends on the temperature and flow velocity of the purge air [19], and the cooling rate of the adsorbent in the cooling stage also depends on the 


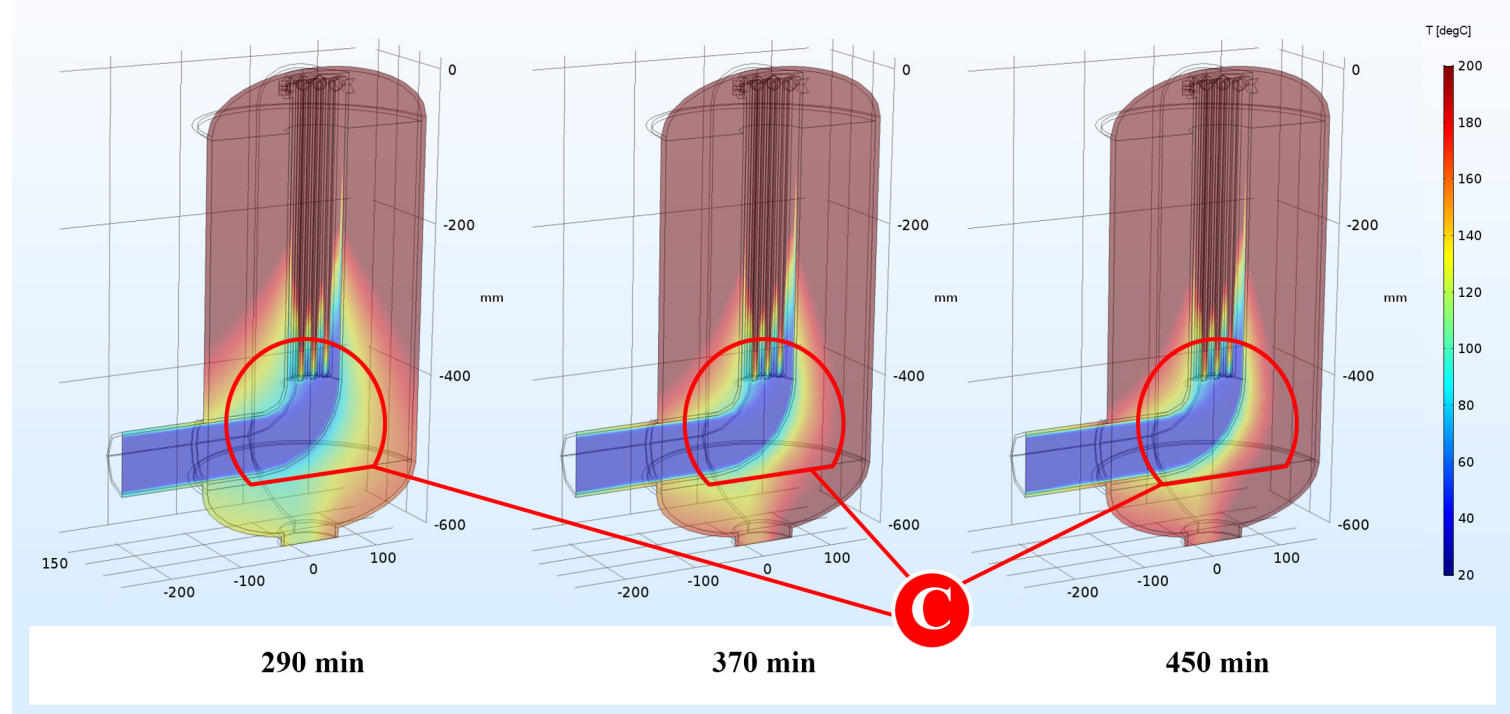

FiguRE 5. The gradient of the temperature of the flowing air, adsorbent, and elements of the adsorption column HT. $\mathrm{C}$ is the zone of reduced intensity of heating of the adsorbent.

cooling air flow rate [20], the presence of stagnation zones indicates that in this region, the process of regeneration of the adsorbent proceeds non-intensively.

\subsubsection{Airflow in the adsorption COLUmn RF}

To conduct a comparative analysis of the flow of air through the RF adsorption column with different deflectors, models of adsorption columns with deflectors of two different types are additionally constructed. In columns of this design, energy is supplied to the volume of the adsorbent using microwave radiation, with no internal heater and internal pipelines. For this reason, the internal geometry of the $\mathrm{RF}$ adsorption column has a classical construction. Thus, the air inflow occurs at the top of the column directly above the volume of the adsorbent (Figure 4, pos. I-II).

During the consideration of the purge air velocity gradient, a zone with a low (up to $0.01 \mathrm{~m} / \mathrm{s}$ ) air velocity in the column with a disk diffuser was also detected (Figure 4. item II, letter B). However, this zone is outside the boundaries of the volume of the adsorbent (Figure 2). Thus, the flow through the volume of the adsorbent in the RF adsorption column, regardless of the inlet diffuser, is more laminar, which provides better conditions for the regeneration of the adsorbent, with other things being equal.

\subsection{OCCURRING OF HEAT TRANSFER PROCESSES IN THE ADSORPTION COLUMN}

The speed of the airflow in the cavity of the column has a direct effect on the dynamics of heat exchange processes. On the basis of the obtained data on the air flow, using the time-dependent solver, the thermal model of the adsorption column was solved.

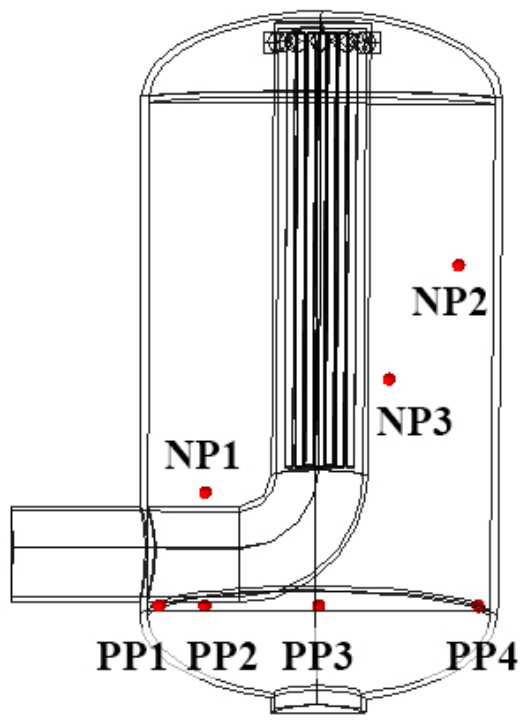

Figure 6. Control points for measuring the temperature in the volume of the adsorbent.

\subsubsection{OCCURRING OF HEAT TRANSFER PROCESSES IN THE ADSORPTION COLUMN HT DURING THE HEATING OF THE ADSORBENT}

The data on the maximum, minimum and average temperatures of the air flowing through the HT adsorption column and through the adsorbent bed during the heating of the adsorbent (Figure 5 ) indicate the presence of a zone of uneven heating of the adsorbent. During the consideration of the temperature gradient, a zone of reduced intensity of adsorbent heating located near the inlet air duct was detected (Figure 5 item $\mathrm{C}$ ). The presence of this zone indicates that in this region of the HT adsorption column, the intensity of the process of regeneration of the adsorbent is 


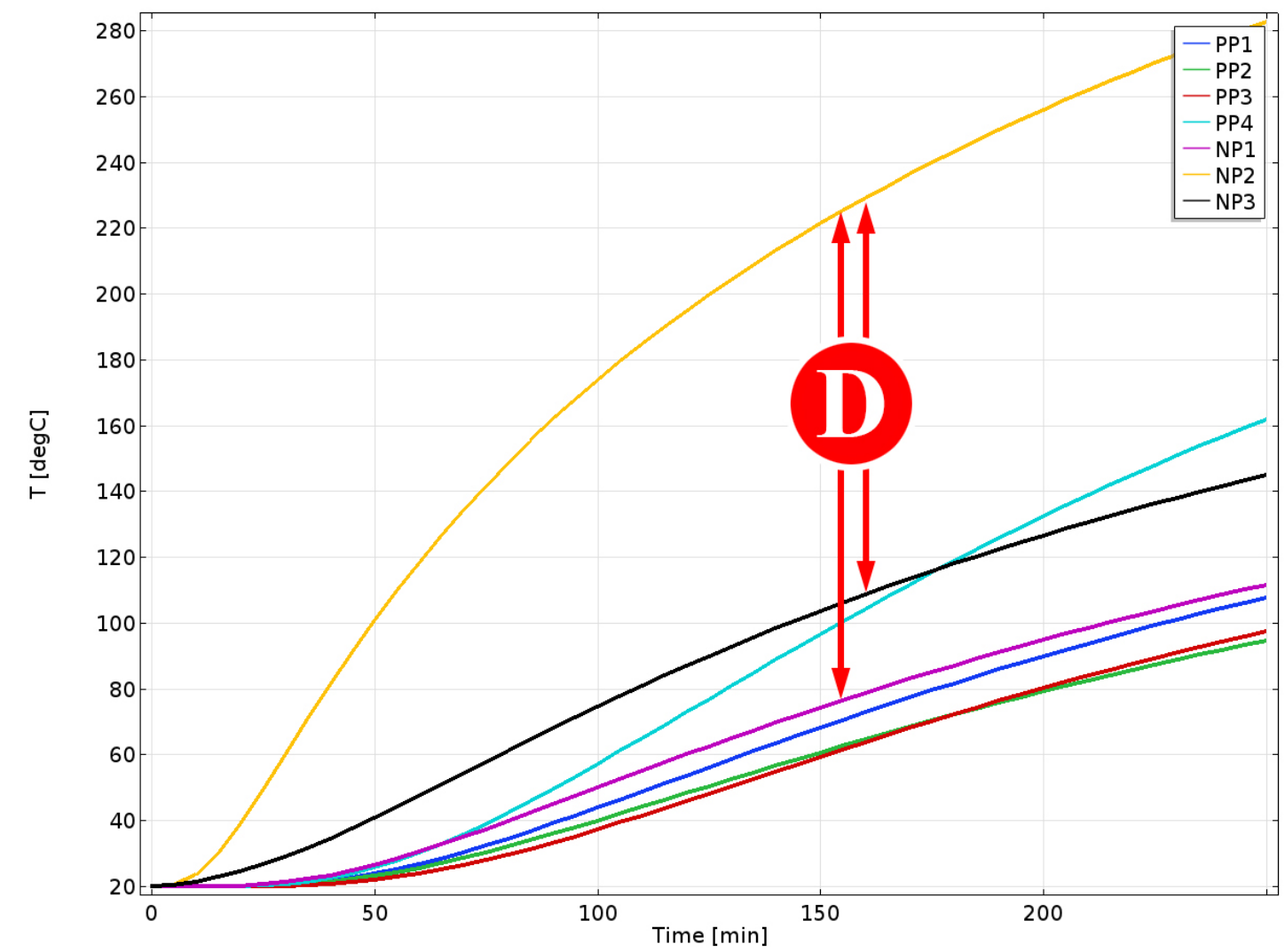

Figure 7. Graph of temperature increase in control points. D is the temperature difference at the point NP2 and NP3, PP4, and also NP1, PP1, PP2, PP3.

lowered. This is due to the cooling of the inlet duct through the unheated air that is on the way to the heater chamber.

The uneven heating of the adsorbent volume is confirmed by the data on the temperature obtained at 7 control points (Figure 6 ) located in the volume of the adsorbent. The control points are arranged in such a way as to quantify the temperature values in that part of the volume of the adsorbent that is located near the inlet duct and near the heater chamber as well as in the laminar air flow area through the adsorbent. This allows us to compare the being obtained values from the point of view of estimating the non-uniformity of the temperature variation of the adsorbent during heating or cooling.

The temperature rise graph in the control points (Figure 7) shows the non-uniformity of the heating of the adsorbent volume in the adsorption column HT. The temperature at a point located at the coordinate where the heated purge air flows uniformly, the point NP2 (Figure 6) shows a stable intense uniform temperature rise, while the temperature rise at the point NP3 is significantly slowed down. The slowdown in the temperature growth at NP3 is due to the proximity of this point to the inlet duct. The air that has not yet been heated by the heater flows in the inlet duct. This causes cooling of this section of the pipeline and the adjacent area of the volume of the adsorbent (Figure 5 letter $\mathrm{C}$ ) due to the cold incom- ing air. At the point $\mathrm{PP} 4$, the temperature increase is also slowed, which is explained by its remoteness from the heat source. However, as the volume of the adsorbent is heated to a temperature of $+150{ }^{\circ} \mathrm{C}$, an intensive temperature increase begins at this point.

The temperature increase at the points PP1, PP2, PP3 is the least intense (Figure 7). This is explained by both the proximity of this points to the inlet duct and by the fact that these points are located in the zone with a low (up to $0.01 \mathrm{~m} / \mathrm{s}$ ) speed of air flow through the volume of the adsorbent (Figure 3, letter A). At the point NP1, which is located above the inlet pipeline, along the route of the cold incoming air, there is also a slow rise in temperature, compared to the point NP2 (Figure 7, letter D), however, more intense than the temperature increase at points PP1, PP2, PP3 (Figure 7, letter D4). This is due to similar reasons, such as the slowdown in temperature growth at the points PP1, PP2, PP3. At the same time, the dynamics of temperature growth at points PP1, PP2, PP3 and NP1 is similar, which is explained by the similarity of occurring processes in the region located near the inlet pipeline (Fig.3, letter A and Figure 5.).

\subsubsection{OCCURRING OF HEAT TRANSFER PROCESSES IN THE HT ADSORPTION COLUMN DURING THE COOLING STAGE OF THE ADSORBENT}

The temperature decrease graph at the control points (Figure 6.) shows the dynamics of temperature decrease in different coordinates of the adsorbent volume 


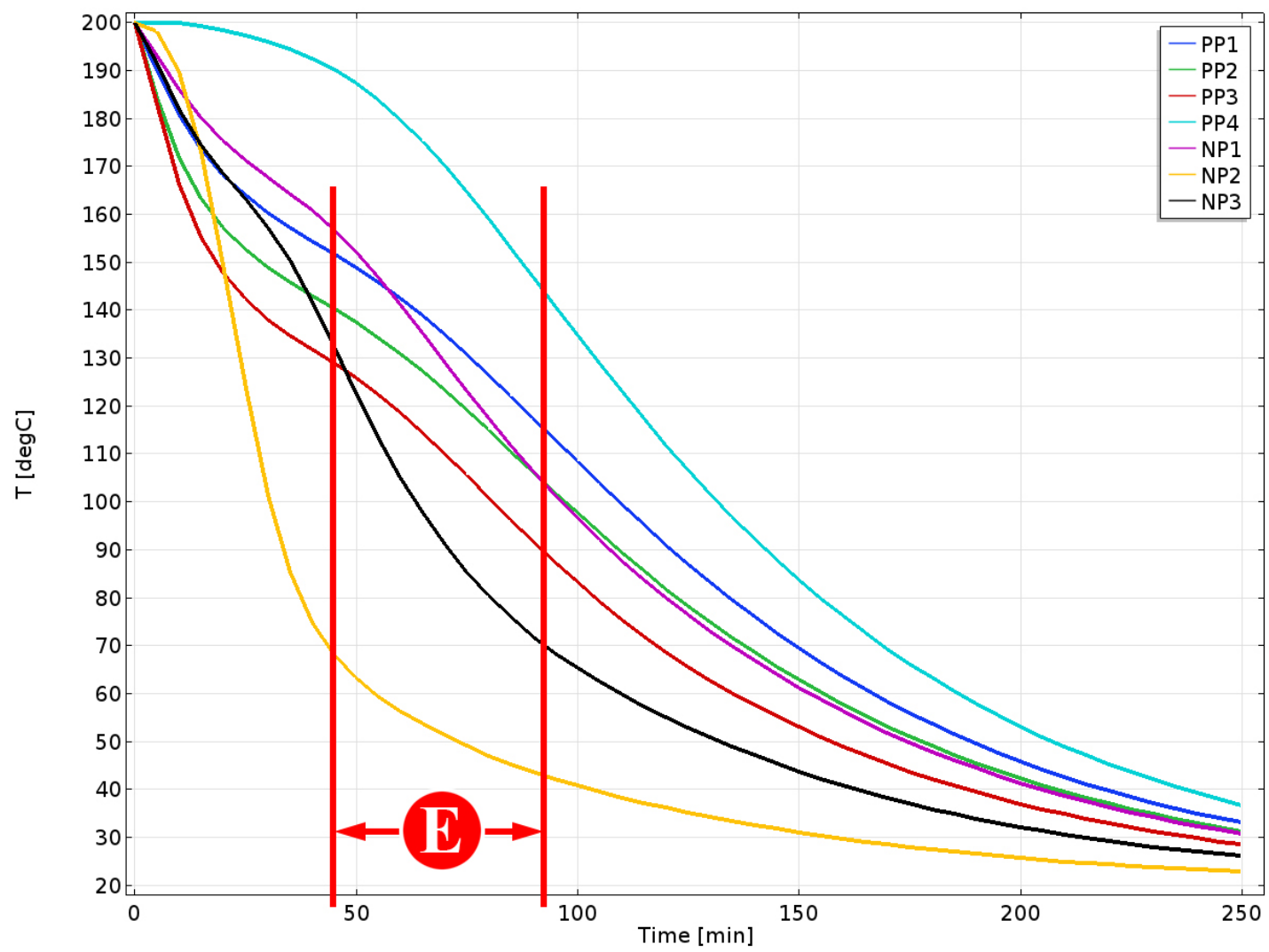

Figure 8. Graph of temperature reduction at control points. E is the time period during which the temperature decrease occurs with acceleration.

in the adsorption column HT (Figure 8). There is a change in the acceleration of the heat transfer during the cooling process. There are three periods with different dynamics of the cooling process. So, in calculating the process of cooling the adsorbent for $250 \mathrm{~min}$, during periods $0-50 \mathrm{~min}$ and $>100 \mathrm{~min}$, a slowing down temperature decrease is observed, as evidenced by the concave shapes of the lines of the temperature change graphs. During a period of 50-90 min, the temperature decreases with a certain acceleration, as evidenced by the convex shapes of the lines of temperature-change graphs.

During the periodic cooling of the adsorbent from the desorption temperature $\left(+200{ }^{\circ} \mathrm{C}\right)$ to the working adsorption temperature $\left(+20^{\circ} \mathrm{C}\right)$, simultaneous periodic cooling of the adsorption column casing in the specified temperature range also occurs. Since the HT adsorption column has a built-in heater, this heater also periodically heats up and then cools down to the working adsorption temperature $\left(+20^{\circ} \mathrm{C}\right)$. This is unavoidable since the purge air at the stage of cooling the adsorbent first flows through the heater chamber before the flow reaches the volume of the adsorbent. During the consideration of the temperature gradient over a period of 50-90 min, this period of time coincides with the completion time of the cooling of the heating element, (Figure 10, letter $\mathrm{H}$ ). Thus, it can be concluded that the thermal energy that is accumulated in the material of the heater and the heater chamber inhibits the growth of the rate of decrease in the temperature of the adsorbent during the stage of cooling the adsorbent to the operating temperature of adsorption. This is because the flow of the cooling air is heated from the heater, which retained the heat after being shutdown, and this heat is transferred to the volume of the adsorbent. Calculations show that it is only when about $85 \%$ of the heat energy of the heater and the chamber has passed to the purge air and, in part, to the adsorbent, the process of cooling the adsorbent begins to accelerate. (Figure 8).

\subsubsection{OCCURRING OF HEAT TRANSFER PROCESSES IN THE RF ADSORPTION COLUMN DURING THE COOLING STAGE OF THE ADSORBENT}

The design of the RF adsorption column, which uses the microwave radiation as the heat source, is characterized by the absence of structural elements in the volume of the adsorbent. Thus, as shown in subparagraph 4.1-2, the flow of air through the adsorbent bed is characterized by a high uniformity and the absence of zones with a low (up to $0.01 \mathrm{~m} / \mathrm{s}$ ) air velocity. At the same time, the temperature front has a curved shape from the walls of the adsorption column to its centre. Cooling of the internal volume of the adsorbent along the vertical axis of the symmetry of the column occurs more intensively. This is due, on the one hand, to the contact of the adsorbent with the heated walls of the adsorption column, which results in conductive heat transfer from the column walls to 


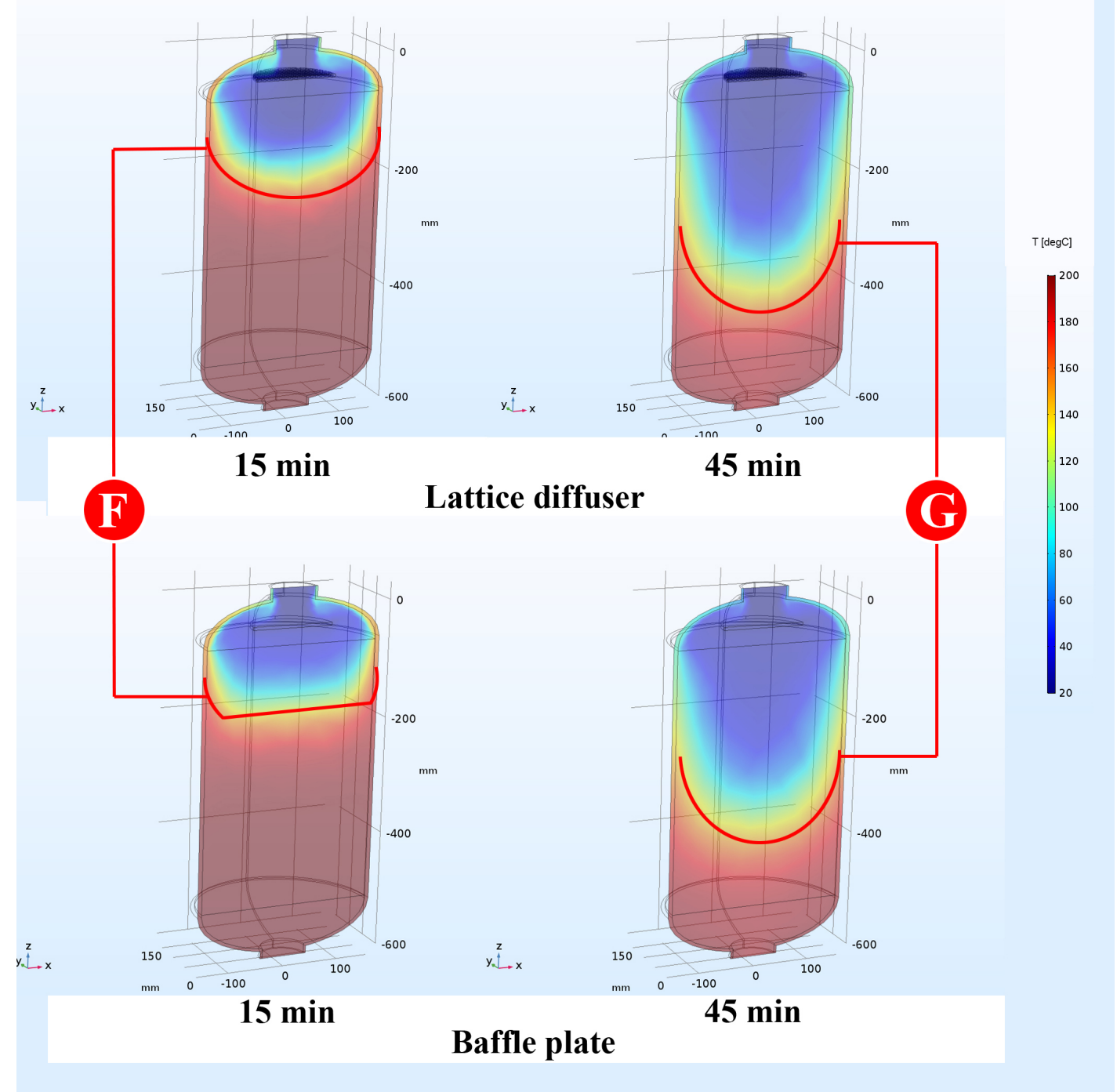

Figure 9. The gradient of the temperature of the adsorbent in the adsorption column RF. F is the difference in the form of the temperature front; $G$ is the identity in the form of a temperature front.

the adsorbent. On the other hand, air that enters the cavity of the RF adsorption column vertically along the axis of symmetry comes to contact mainly with the area of the adsorbent volume near the vertical axis of symmetry of the column (Figure 10.

In order to achieve better uniformity of cooling of the adsorbent volume, the influence of the internal elements of the RF adsorption tower on the character of temperature change in the volume of the adsorbent was investigated.

As an internal element contributing to a flatter temperature front, which indicates a greater uniformity of temperature in the horizontal layers of the adsorbent, lattice diffuser and baffle plate are used.

The study shows that the use of a shield baffle plate provides a flatter temperature front, compared to a lattice diffuser (Figure 9 letter F). This is maintained for 20 min during the purge process. Further, the form of the temperature front acquires a concave character and, by $40 \mathrm{~min}$ of the blowing process, the difference in the use of the lattice diffuser and baffle plate is levelled (Figure 9 , letter $\mathrm{G}$ ).

It should be noted the appearance of a zone with a low value of the air flow rate in the case of using a baffle plate, but the location of this zone outside the volume of the adsorbent (Figure 4 letter B) does not adversely affect the heating and cooling of the adsorbent.

\subsubsection{Comparative AnAlysis of the COURSE OF} HEAT EXCHANGE PROCESSES IN THE STAGE OF COOLING OF THE ADSORBENT IN THE HT ADSORPTION COLUMN AND RF ADSORPTION COLUMN

In the process of solving the thermal model of the adsorption column HT, and the adsorption column RF, data were obtained on the maximum, minimum and average temperatures in the volume of the adsorbent material that these columns contain. The calculation of these values and a comparative analysis of their changes are carried out. 


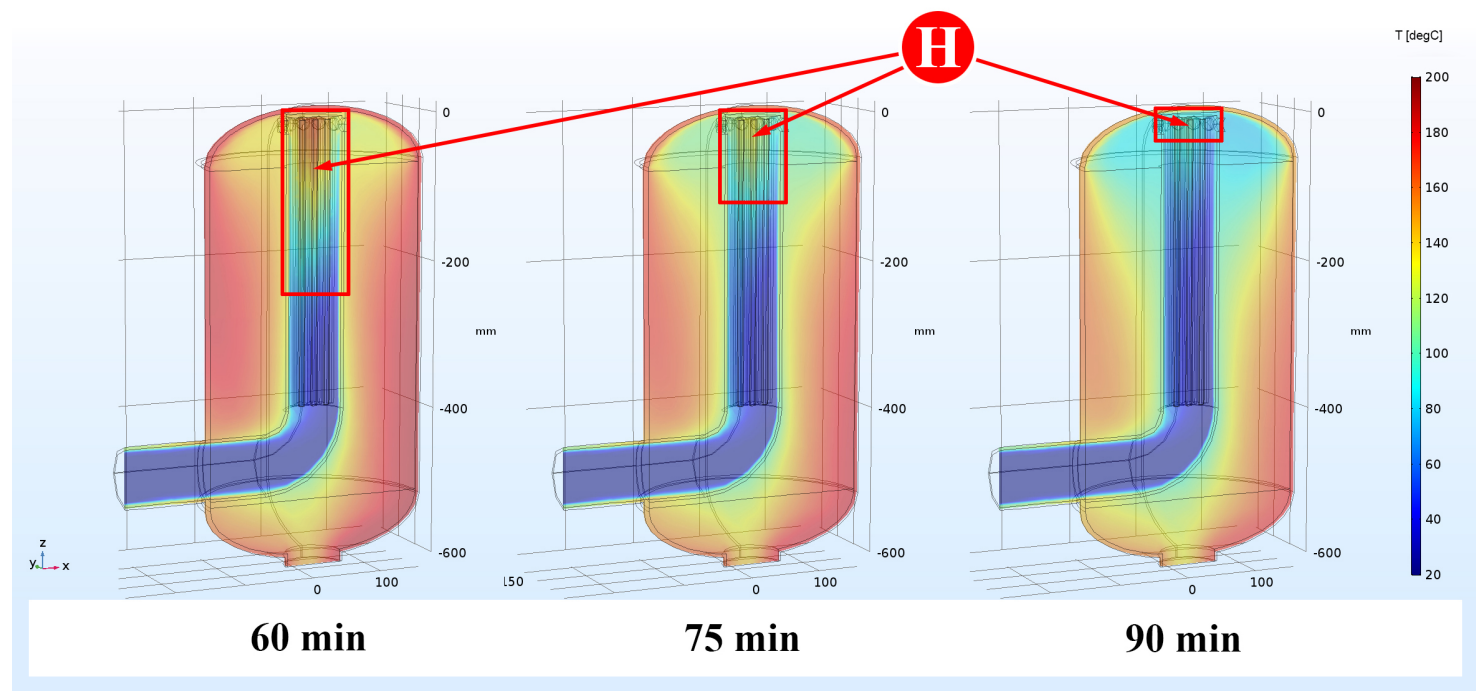

Figure 10. Gradients of the temperature value of the flowing air, adsorbent, and elements of the HT adsorption column with the internal heater. $\mathrm{H}$ is the temperature gradient of the not yet cooled part of the heating element.

On the comparative graph of the decrease in the temperature of the adsorbent volume and the elements of the adsorption columns of the HT and RF structure (Figure 11), the dynamics of the change in the maximum, minimum and average temperatures are shown. The graph clearly shows the lower intensity of the process of cooling the adsorbent in the adsorption column HT, compared with the intensity of this process in the adsorption column RF.

There is a sharp drop in the minimum temperature to almost $+20^{\circ} \mathrm{C}$ immediately at the start of the purge of the RF column (Figure 11, Min $T$ for RF). The lowering of the minimum temperature with the start of the HT purge is more gradual, due to the fact that the purge air passes through the chamber with a warm heater, heats up, and has a temperature above + $20^{\circ} \mathrm{C}$. For the same reason, when the blowdown starts, the higher maximum temperature remains longer and has a higher value (Figure 11, Max $T$ for HT). It is noted that as the cooling process proceeds for more than $150 \mathrm{~min}$, the dynamics of the processes at all observed points become similar. This is due to the fact that the built-in heater, heater chamber and internal elements of the HT column have already cooled to a temperature of $+50{ }^{\circ} \mathrm{C}$ and does not affect the dynamics of the process.

In general, it should be noted that the cooling process of the HT adsorption column, other things being equal, is less dynamic than the process of cooling the $\mathrm{RF}$ column, and the mean temperature value is higher (Figure 11, Average $T$ ).

The calculation shows that the inevitable presence of internal structural elements in the cavity of the HT adsorption column leads to an increase in energy costs, which, inevitably, is spent on cooling the volume of the adsorbent to the adsorption temperature. This is due to the periodic accumulation of thermal energy in the heater and by the heater chamber during the heating of the adsorbent to a temperature of $+200^{\circ} \mathrm{C}$ during the regeneration stage.

\section{Conclusions}

The use of an internal heater in the construction of the adsorption column leads to the appearance of undesirable factors from the point of view of the regeneration process of the adsorbent. Based on the results of a study of the HT adsorption column by computer modelling, it is possible to note such undesirable phenomena as the appearance of zones with low air velocities (up to $0.01 \mathrm{~m} / \mathrm{s}$ ), uneven heating and cooling of the adsorbent, and a greater heat capacity of the adsorption column, compared with the RF adsorption column. While the design of the adsorption dryer, which has the adsorption columns with the internal heater, has some advantages, in terms of minimizing the heat losses during the heat transportation, these factors have a negative effect on the process of regenerating the adsorbent.

The use of microwave radiation to regenerate the adsorbent helps to reduce the thermal losses of the dryer by reducing the heat capacity of the previously heated elements of the structure. This reduces the loss of the purge air for regeneration [21. Taking into account the fact that the RF-regeneration of the adsorbent occurs at a lower temperature [18, the purge air costs will be even less. So, the technology of regenerating the adsorbent using the microwave energy is more economical.

The design of the adsorption desiccant, which uses the microwave radiation as a heat source, is more appropriate. In this case, the problem of heat loss during its transfer using a purge air flow can be effectively solved using microwave radiation. This involves heating the volume of the adsorbent directly in the 


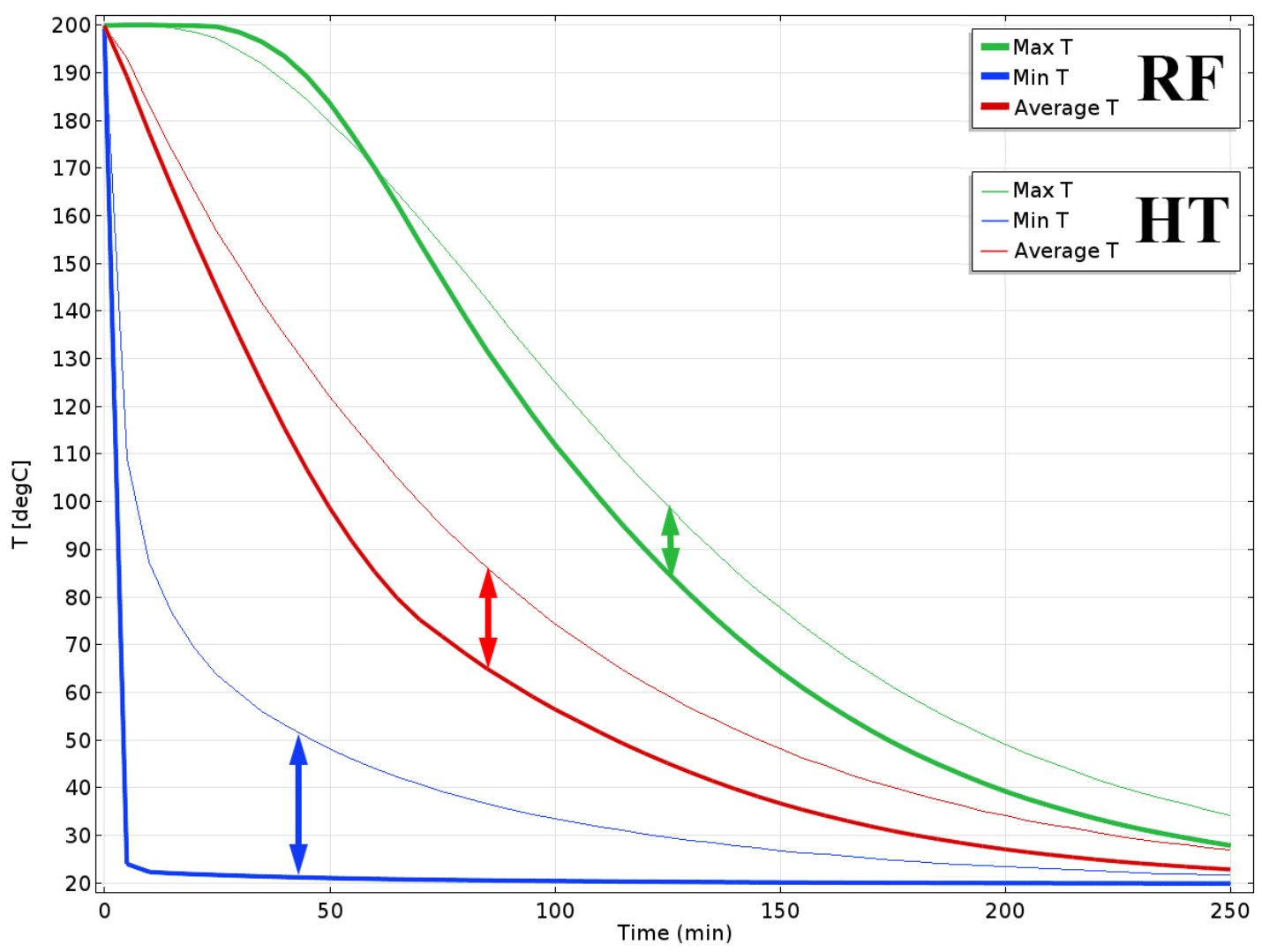

Figure 11. The graph of the decrease in the maximum $(\operatorname{Max} T)$, minimum $(\operatorname{Min} T)$ and average (Average $T)$ temperature in adsorption columns of the RF and HT type.

cavity of the adsorption column [22 24$]$, as in the case of an internal heater.

The use of microwave radiation for the purpose of heating the adsorbent during its regeneration can effectively combine the advantages in minimizing the heat losses of the dryer, the best conditions for purging the adsorbent by flowing air from the point of view of hydrodynamics, and to minimize the heat capacity of the adsorption column structure.

\section{REFERENCES}

[1] Karpus V.E., Ivanov V.A. Choice of optimal configuration of modular reusable fixtures. Russian Engineering Research, Vol. 32, Issue 3, 2012, pp. 213 -219, DOI:10.3103/S1068798X12030124

[2] Karpus V., Ivanov V., Dehtiarov I. et al. Technological assurance of complex parts manufacturing. In: Ivanov V. et al. (eds.) Advances in Design, Simulation and Manufacturing. DSMIE-2018. Lecture Notes in Mechanical Engineering, 2019, pp. 51 -61, DOI:10.1007/978-3-319-93587-4_6

[3] Fesenko, A., Yevsiukova, F., Basova, Y., Ivanova, M. and Ivanov, V.: Prospects of Using Hydrodynamic Cavitation for Enhancement of Efficiency of Fluid Working Medium Preparation Technologies, Periodica Polytechnica Mechanical Engineering, 62(4), 2018, pp. 269-276. doi: DOI:10.3311/PPme.11877

[4] Skriabyn, V.I.: The course of lectures on heating engineering [in Russian]. XuMuK - chemistry site. Electronic resource [2018-03-24]. http://www . xumuk.ru/teplotehnika/054.html
[5] Dobrotvorskiy, S., Dobrovolska, L., Aleksenko B.: Computer simulation of the process of regenerating the adsorbent using microwave radiation in compressed air dryers. Lecture Notes in Mechanical Engineering: Springer, 2017, p. 511-519. 2017. DOI:10.1007/978-3-319-68619-6_49

[6] Farooq, S., Ruthven, D.M.: Heat Effects in Adsorption Column Dynamics. 1. Comparison of One and Two-Dimensional Models: Industrial \& Engineering Chemistry Research. 29(6), 1990, p. 1076-1084. DOI:10.1021/ie00102a019

[7] Auerbach, Scott M., Carrado, Kathleen A., Dutta, Prabir K.: Handbook of Zeolite Science and Technology. New Yorc-Basel: Marcel Dekker, Inc., 2003.

[8] Atlas Copco. Heat Reactivated Adsorption Air Dryers. Direct industry. Electronic resource [2018-06-12]. http://pdf.directindustry.com/pdf/atlas-copcocompressors/bd-100-3000/5575-116858.html

[9] Silica - Silicon Dioxide ( $\mathrm{SiO} 2)$. Azo Materials. Electronic resource [2018-06-12]. http: //www.azom.com/properties.aspx?ArticleID=1114

[10] Ng, K.C., Chua, H.T., Chung, C.Y., Loke, C.H., Kashiwagi, T., Akisava, A., Saha, B.B.: Experimental investigation of the silica gel-water adsorption isotherm characteristics: Applied Thermal Engineering, Elsevier, 21(16), 2001, p. 1631-1642. DOI:10.1016/S1359-4311(01)00039-4

[11] Dobrotvorskiy S., Dobrovolska L., Aleksenko B., Basova Y.: The Use of Waveguides with Internal Dissectors in the Process of Regeneration of Industrial 
Adsorbents by Means of the Energy of UltrahighFrequency Radiation. In: Ivanov V. et al. (eds) Advances in Design, Simulation and Manufacturing. DSMIE 2018. - Held during June 16, 2018. Lecture Notes in Mechanical Engineering. Springer, Cham, 2019, p. 433-442. DOI:10.1007/978-3-319-93587-4_45

[12] Hoseinzadeh, S. and Azadi R. Simulation and optimization of a solar-assisted heating and cooling system for a house in Northern of Iran: Renewable Sustainable Energy, American Institute of Physics. 9 (4), 045101-13, 2017. DOI:10.1063/1.5000288

[13] Ghasemiasl, R., Hoseinzadeh, S., Javadi M. A.: Numerical analysis of energy storage systems using phase-change materials with nanoparticles: Journal of Thermophysics and Heat Transfer. vol 32, No 2, 2018, p. 440-448. DOI:10.2514/1.T5252

[14] Hoseinzadeh, S.,Azadi R. J.: Simulation and optimization of a solar-assisted heating and cooling system for a house in Northern of Iran. J. Renewable Sustainable Energy, 9:4., 2017, 045101. DOI: $10.1063 / 1.5000288$

[15] Hoseinzadeh S., Ghasemiasl R., Havaei D., Chamkha A.J.: Numerical investigation of rectangular thermal energy storage units with multiple phase change materials, Journal of Molecular Liquids, Volume 271, 2018, pp. 655-660

[16] Dordevic, N., Salamin, Ya. P\&S Comsol. Design Tool Week 3: EM Review. Electronic resource [2018-06-14]. http://people.ee.ethz.ch/ fieldcom/pps-comsol/ documents/Presentation/Lecture2_IntroCOMSOL.pdf

[17] Lykov, A.W.: Theory of Drying. Moscow: Energia, 1968. [Russian]

[18] Dobrotvorskiy S., Aleksenko B., Dobrovolska L., Basova Ye.: Effect of the application of microwave energy on the regeneration of the adsorbent: Acta Polytechnica, Czech Technical University in Prague. 58(4), 2018, pp. 217-225. DOI:10.14311/AP.2018.58.0217
[19] Datta, A.K.: Porous media approaches to studying simultaneous heat and mass transfer in food processes. I: Problem formulations: Journal of Food Engineering, vol. 80 , issue 1,2007 , p. 80-95. DOI:10.1016/j.jfoodeng.2006.05.013

[20] Datta, A.K.: Porous media approaches to studying simultaneous heat and mass transfer in food processes. II: Property data and representative results: Journal of Food Engineering, vol. 80, issue 1, 2007, p. 96-110. DOI: 10.1016/j.jfoodeng.2006.05.012

[21] White, D.: Regenerative Desiccant Compressed Air Dryers. Poultry Audit \& Air Quality: Compressed Air Best Practices, 12(1), 2015, p. 26-31. Electronic resource [2017-12-12] https://www . airbestpractices . com/technology/air-treatmentn2/regenerativedesiccant-compressed-air-dryers

[22] Fedyunin, P.A.: Microwave thermowomagometry. Moscow: Publishing house Mechanical Engineering-1, 2004. [Russian]

[23] Orfanidis, Sophocles J.: Electromagnetic Waves and Antennas. Electronic resource [2018-06-12]. http://eceweb1.rutgers.edu/ orfanidi/ewa/

[24] ECE-329 Fields and Waves I.3. Monochromatic waves and phasor notation. Phasor form of Maxwell's equations and damped waves in conducting media: Lecture Notes. ECE ILLINOIS Department of Electrical and Computer Engineering. Electronic resource [2017-02-10]. http:// jsa.ece.illinois.edu/ece329/notes/329lect21.pdf

[25] Shevchenko O.A., Suris A.L., Shatalov A.L.: Study of the process of regeneration of silica gel by the method of its drying in a microwave electromagnetic field Izvestiya MGTU, 3(17), 2013. Electronic resource [2018-10-10] https://cyberleninka.ru/article/n/issledovanieprotsessa-regeneratsii-silikagelya-metodom-egosushki-v-sverhvysokochastotnomelektromagnitnom-pole

[26] E. R. (Ross) Crain, P.Eng.Career History: "Crain's Petrophysical Handbook, 2018. Electronic resource [2017-02-10] www.spec2000.net. 